\title{
Detection and Tracking of Infrared Dim Small Image Sequence Moving Target
}

\author{
Huanhai Yang*
}

Shandong Institute of Business and Technology, Yantai, Shandong 264005, China

\begin{abstract}
Infrared dim target detection and tracking is widely used in precision guidance, automatic control and other fields. As the small target imaging system has far distance, small area, low SNR infrared characteristics and undergoes image preprocessing, dim target detection technology is more important and more difficult. As a full play of the advantages of infrared small target detection technique has been provided, this has significance in infrared imaging. Based on introducing the tracking technology development status at home and abroad by the study of small target detection, the problems of detecting and tracking small moving target in infrared image sequence of background noise and interference conditions, aiming at dynamic planning in energy diffusion problem are presented. The improved algorithm of dynamic programming is also presented including weighted dynamic programming algorithm and the realization steps of the algorithm. Detection structure and the experimental results are analyzed. The results show that the algorithm has a good effect. In the end, this paper studies the rejected false targets in IR image track association method to obtain real object trajectories for moving small target detection, so as to improve the detection rate and reduce the false alarm rate.
\end{abstract}

Keywords: Local spectrum suppression, non-separable wavelet, score fusion, target enhancement.

\section{INTRODUCTION}

The requirements of modern high technology war weapon system include a fast response and good stealth performance that can timely detect the target, track the target, capture and lock the target, and realize the effective attack [1-6]. The infrared detection and imaging distance tracking technology can well satisfy this condition as it possesses conventional imaging modalities such as visible light imaging and radar imaging compared. Moreover, it has the following advantages: better environmental adaptability to visible light, especially at night and work ability even in harsh climate; good hidden performance, generally passive acceptance of the information of the target; the ratio of radar and laser detector security and confidentiality, which is not easily disturbed and discovered; compared with radar, its infrared wavelength is short, can obtain the target image with high resolution and is more suitable for the target key parts (point of impact) choice [7].

With the increase of application and detection distance of various infrared stealth technology, when the imaging system and the target distance are far, even if the target itself is very big, the projection in the image plane becomes a small target, with size only a few pixels, or even a pixel [8-11]. Because of the requirements of the role of distance, target with low SNR than the average gray level of clutter is sometimes even higher than the target gray. At the same time, this kind of application has multi requirements of target detection with real-time and low false alarm probability. This provides

*Address correspondence to this author at the Shandong Institute of Business and Technology, Yantai, Shandong 264005, China;

Tel: 18955169345; E-mail: Huanhai Yang@163.com a dim target detection, causing difficulty in recognition and tracking [12].

This paper studies wavelet transform infrared image preprocessing method based on multi resolution analysis of wavelet transform. The characteristics of suitable small target infrared image preprocessing, image preprocessing after background clutter suppression and signal to noise ratio are observed to be improved. Based on the research background in infrared image sequences generated with target detection algorithm based on Kalman filter, the principle of the algorithm is given. Background generation method in time domain of the fading recursive least squares method and moving object extraction method has been used in the experiment. The results show that the algorithm can detect small target in infrared image target having better effect. On the basis of pretreatment of small moving target continuity characteristics, the sequence image motion of small target detection algorithm is studied based on weighted dynamic programming while providing a dynamic programming (DP) algorithm principle and analyzing the direct gray adding method and dynamic programming based on the likelihood function (DP) algorithm.

\section{RELATED IMAGE THEORY AND RESEARCH STATUS}

\subsection{Current Situation of Infrared Small Target Detection}

Small infrared target detection and tracking is one of the most challenging issues. From the beginning of 1989 [1316], SPIE organizes a "small target signal and data processing" international conference every year for conducting small target detection of new technology exchange. The 
United States [17] in the 20 century 50s and 60s began infrared search and tracking technology research work, which was followed by France, Germany, Russia, Canada and other countries carrying out the research work [18]. At present, foreign research on "passive infrared early warning system" technology to increase investment, especially in the United States is an important task for national security defense system. "Passive infrared early warning detection system research" technology has also promoted the research work on the subject of "detection of infrared dim small target". Although, the research on the subject has begun recently at home, but from the published papers, it can be seen that the research is being conducted similar to the foreign level with all kinds of new ideas. Moreover, new algorithm has been put forward, and the theory system of infrared dim target detection has been enriched and perfected $[19,20]$.

According to the different angles, the detection algorithm can have different classification methods: the different information used can be divided into direct method and indirect method; the detection and tracking of sequence can be divided into different detections before tracking method (DBT) and track before detect method (TBD); and also a classification method of spatial filtering and time filtering and static background and moving background classification method [21]. The following classifications according to the direct method and indirect method are introduced and a brief analysis of the existing detection algorithms is conducted in this paper [22].

The direct method of directly using the gray values of pixels was detected. The flow detection algorithms can be divided into three parts: first, background estimation, obtaining the estimation of image; segmentation according to the difference between the original image and the background image and then obtaining the candidate target points; finally, making use of the target's moving character, removing high frequency noise points to obtain the real target [23, 24].

\subsection{The Basic Principle of Sparse Representation}

The number of vector elements contained are called vector dimension, an $\mathrm{M}$ dimensional vector by $\mathrm{M}$ elements, with each element of the vector corresponding to the value of the dimensions, namely $\varphi=\left\{\varphi_{i}\right\}, i=1,2, \ldots, M, . \mathrm{T}$ linearly independent vectors $\Psi=\left\{\varphi_{i}\right\}, i=1,2, \ldots, T$, can be composed of a set of base and can use linear combinations representing other vector in this space. For any $\mathrm{M}$ dimensional vector space, when $\mathrm{T}<\mathrm{M}$, the linear combination can be only a part of the representation of space vector, which is called underdetermined, or is incomplete. But when $\mathrm{T}=\mathrm{M}$, any vector $\mathrm{s}$ of $\mathrm{M}$ dimensional vector space can only use linear combinations to represent:

$s=\sum_{i} a_{i} \varphi_{i}, i=1,2, \ldots, M$

Because of the infinite matrix being at full rank, any vector s only carries on the decomposition, and is uniquely determined by the factorization results obtained. Usually the type (2) is written in matrix form:

$$
s=\left[\begin{array}{llll}
\varphi_{1} & \varphi_{2} & \ldots & \varphi_{M}
\end{array}\right]\left[\begin{array}{c}
a_{1} \\
a_{2} \\
\vdots \\
a_{M}
\end{array}\right]=\Psi a
$$

\section{ADAPTIVE INFRARED SMALL TARGET DETEC- TION FOR OVER COMPLETE SPARSE REPRESEN- TATION OF IMAGE}

\subsection{The Infrared Small Target Image Model}

Given a digital infrared image $f(x, y)$ of size $M \times N$, its transformed form in the frequency domain is:

$$
F(u, v)=\frac{1}{M N} \sum_{x=0}^{M-1} \sum_{y=0}^{N-1} f(x, y) \exp (-j 2 \pi(u x / M+v y / N))
$$

As in the 1-D case, (2) must be the evaluated values of the discrete variables $u$ and $v$ in the ranges $u=0,1,2, \ldots, M-1$ and $v=0,1,2, \ldots, N-1$. Given the transform $F(u, v), f(x, y)$ can be obtained by using the inverse discrete Fourier transform:

$$
f(x, y)=\frac{1}{M N} \sum_{u=0}^{M-1} \sum_{v=0}^{N-1} F(u, v) \exp (j 2 \pi(u x / M+v y / N))
$$

In general, the components of the Fourier transform are complex quantities. As in the analysis of complex numbers, it is more convenient to express $F(u, v)$ in polar coordinates:

$$
F(u, v)=|A(u, v)| \exp (-j P(u, v))
$$

where its magnitude $A(u, v)$, namely the amplitude spectrum of the Fourier transform can be expressed by,

$$
A(u, v)=\left[R^{2}(u, v)+I^{2}(u, v)\right]^{1 / 2}
$$

And $P(u, v)$, called the phase spectrum of the transform is defined as:

$P(u, v)=\arctan \left[\frac{I(u, v)}{R(u, v)}\right]$

Both $A(u, v)$ and $P(u, v)$ are the real and imaginary parts of $F(u, v)$, respectively.

Because the zero-frequency term dominates the values of the amplitude spectrum, the dynamic range of other intensities in the displayed image is compressed. To bring out those details, a log transformation is carried out. The log amplitude spectrum is obtained by:

$$
L(u, v)=\log (A(u, v))
$$

For a better illustration of the above amplitude spectrum, Fig. (1) (a) shows an original infrared image. Fig. (1) (b) shows the amplitude spectrum. 


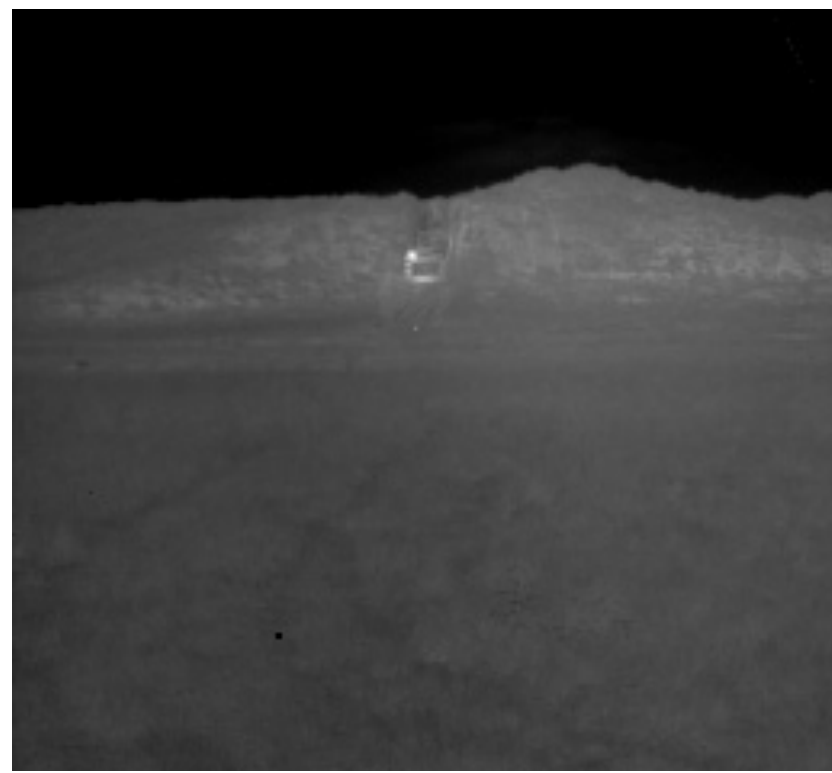

(a) The infrared image

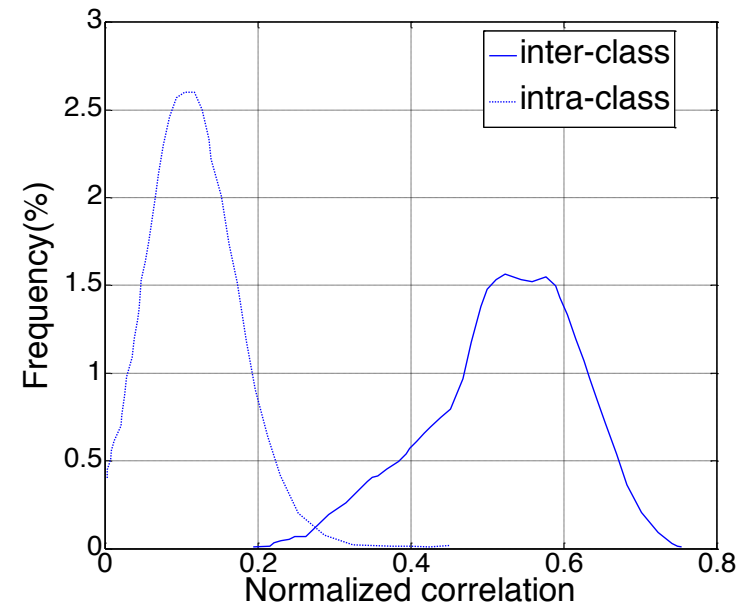

(b) The amplitude spectrum

Fig. (1). The original infrared image and its log amplitude spectrum.

Dim small target in image sequences detection and tracking is one of the key technologies of detection and tracking system of imaging. The related technology which is involved mainly includes image preprocessing, dim small target detection and tracking small target in three aspects. For the infrared imaging target detection system, usually the target is always hidden in the strong background. In this case, if the direct detection of target is very difficult, the most feasible approach is to use some method of signal processing, removing the background interference in target detection, namely, image preprocessing. Image preprocessing is the precondition and basis of target detection and tracking algorithm, with its performance directly affecting the performance of target detection and tracking.

\subsection{Log Amplitude Spectrum Calculation}

The endpoint detection is from a section of speech signal in the detection of the starting point and ending point of speech, deleting the silent section, in order to reduce the calculation amount of feature extraction and accuracy, shorten the training and recognition time system, and improve the recognition rate. The traditional endpoint detection method is used for obtaining image signal short-time energy and shorttime zero crossing rate because of the short time energy point of view, maximum imaged signal and minimum sound signal of silent period.

Suppose an image over frame processing after the $\mathrm{N}$ frame signal for Xn (m), its length is denoted by $\mathrm{N}$, defined by short-time energy and short-time zero crossing rate: 

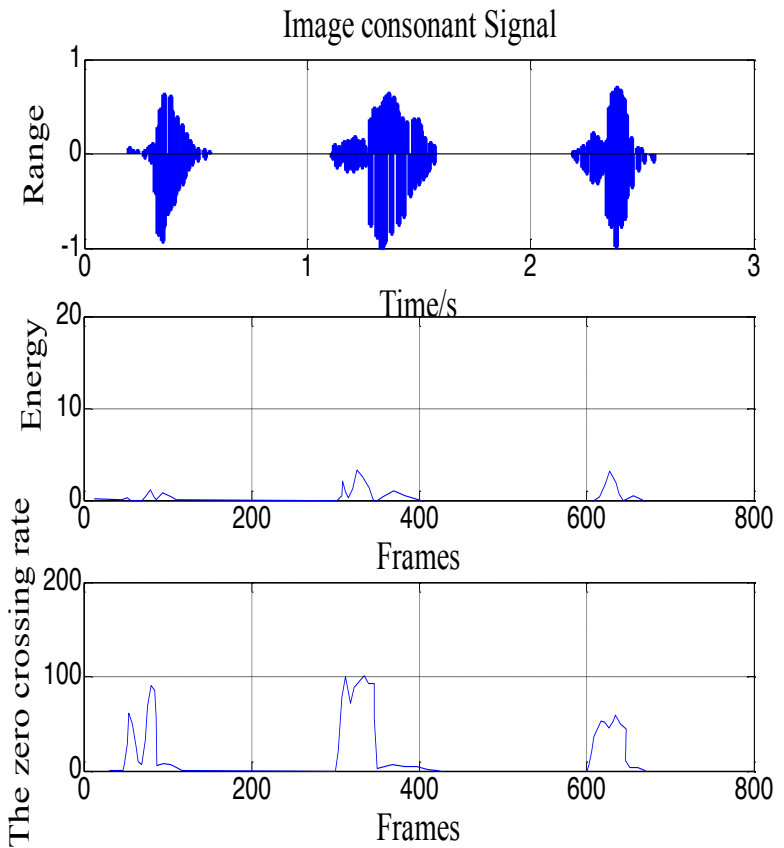

Fig. (2). Section of imaged signal and the image signal short-time energy.

$E_{n}=\sum_{m=0}^{N-1} x_{n}^{2}(m)$

The short-time zero crossing rate of $\mathrm{Zn}$ is:

$Z_{n}=\frac{1}{2 N} \sum_{m=0}^{N-1}\left|\operatorname{sgn}\left[x_{n}(m)\right]-\operatorname{sgn}\left[x_{n}(m-1)\right]\right|$

Sgn[] is the sign function, the expression for which:

$\operatorname{sgn}(x)= \begin{cases}1, & x \geq 0 \\ -1, & x<0\end{cases}$

Fig. (2) shows a section of imaged signal and the image signal short-time energy and short-time zero crossing rate:

\section{EXPERIMENTAL RESULTS}

Fig. (3) shows the experimental results of multilevel filter method: Fig. (3) (a) is the original image containing target part; graph (b) is the result of the first stage low-pass filter of the results after Lp1 graph: graph (c) is a graph; (a) and (b) is the difference chart diagram. From graph (c), it can be seen that most of the background interference is filtered by the residual noise and the target. Diagram (d) is a graph (b) of the results of the second stage low-pass filter after the Lp2 graph; graph (E) is a graph (a) and map (d) is the difference map which can be seen from the graph two times after filtering with suppressed background signal and enhanced target and noise. Diagram (f) is a graph (e) through which the results of the third stage low-pass filter after the map, when the noise is suppressed and the target is enhanced. Visible, multilevel filter for infrared small target image that meets the above conditions can effectively suppress noise and back- ground, enhancing the contrast of the target, which is convenient for small target detection and tracking follow-up.

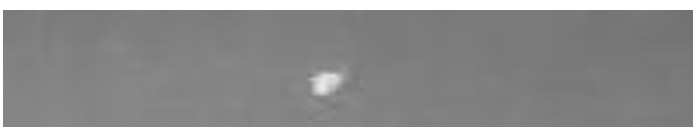

(a) Origin image

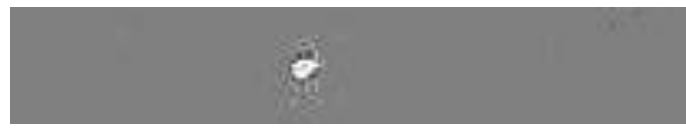

(b) LP1 filtering image

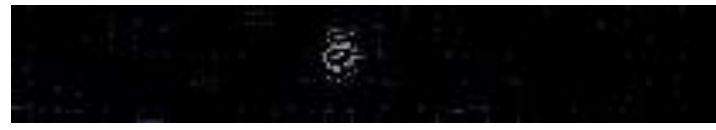

(c) LP1 difference image

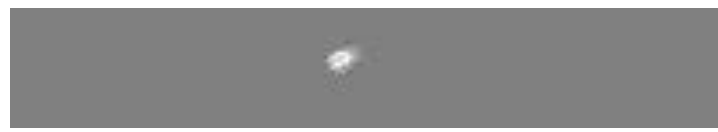

(d) LP2 filtering image

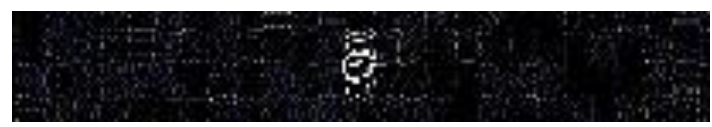

(e) LP2 difference image

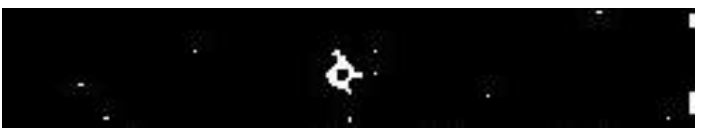

(f) LQ difference image

Fig. (3). The experimental results of multilevel filter method. 


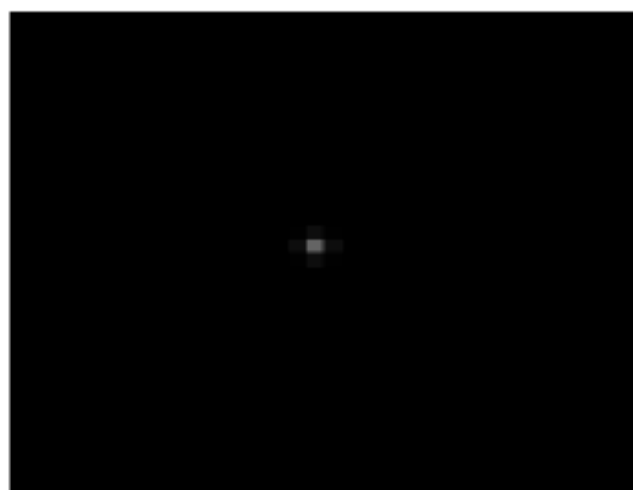

Fig. (4). The image with the infrared small target.

Gauss gray model was first proposed by Chan. The simple point source target using two-dimensional Gauss distribution has the mathematical formula as:

$$
I(i, j)=I_{m} \exp \left(-\frac{1}{2}\left[\frac{\left(i-x_{0}\right)^{2}}{\sigma_{x}^{2}}+\frac{\left(j-y_{0}\right)^{2}}{\sigma_{y}^{2}}\right]\right)
$$

In the formula, Im is the maximum gray value at the center of the target, the maximum brightness deciding target; $\sigma$ $\mathrm{X}$ and $\sigma \mathrm{Y}$ levels are attenuation parameters and vertical attenuation parameters, determining the target pixel value attenuation characteristics; $(\mathrm{X} 0, \mathrm{Y} 0)$ is the center position for the target in infrared image; $(i, j)$ is the coordinate of the value of each pixel in infrared image. The figure below shows the use of Gauss gray model. The pixel near infrared small target model and the center of the generated values has $\mathrm{Im}=100$, with $\sigma \mathrm{X}$ and $\sigma \mathrm{y}$ being $0.5,0.5$. Fig. (4) shows the image with the infrared small target and the value of gray scale image center Table $\mathbf{1}$.

Table 1. The value of gray scale image center.

\begin{tabular}{|c|c|c|c|c|}
\hline $5.2382 \mathrm{e}-10$ & $2.1324 \mathrm{e}-7$ & $1.6321 \mathrm{e}-6$ & $2.1324 \mathrm{e}-7$ & $5.2382 \mathrm{e}-10$ \\
\hline $1.3362 \mathrm{e}-05$ & 0.0058 & 0.0326 & 0.0058 & $1.3362 \mathrm{e}-05$ \\
\hline 0.0058 & 1.6257 & 12.3548 & 1.6257 & 0.0058 \\
\hline 0.0326 & 12.3548 & 100 & 12.3548 & 0.0326 \\
\hline 0.0058 & 1.6257 & 12.3548 & 1.6257 & 0.0058 \\
\hline $1.3362 \mathrm{e}-05$ & 0.0058 & 0.0326 & 0.0058 & $1.3362 \mathrm{e}-05$ \\
\hline $5.2382 \mathrm{e}-10$ & $2.1324 \mathrm{e}-7$ & $1.6321 \mathrm{e}-6$ & $2.1324 \mathrm{e}-7$ & $5.2382 \mathrm{e}-10$ \\
\hline
\end{tabular}

From the graph, it can easily be seen that Gauss gray model has shortcomings: attenuation is too sharp, causing the target edge present a saw tooth shape, but the actual imaging infrared small target is generally relatively smooth edge. Therefore, the need for Gauss gray level attenuation model parameters and vertical attenuation $\sigma \mathrm{x}$ and $\sigma \mathrm{y}$ respectively make the corresponding constraint. When Im is large, $\mathrm{X}$ and sigma y should also take larger values but when Im is low, $\mathrm{X}$ and the $\sigma \mathrm{y}$ should also take a small value. The ratio of $\mathrm{Im}$ and $\mathrm{X}$ and $\sigma \mathrm{y}$ is within a certain range, which is the improved Gauss gray model:
The value of the constraint parameter T1 and T2 is decided by experience. The figure below shows the pixel near infrared small target model and center Gauss gray model generated using the improved value, including $\operatorname{Im}=100, X$ and the $\mathrm{Y}$ as $0.5,0.5, \mathrm{~T} 1=20, \mathrm{~T} 2=60$. Fig. (5) shows the image with the infrared small target and the value of gray scale image center Table 2 .

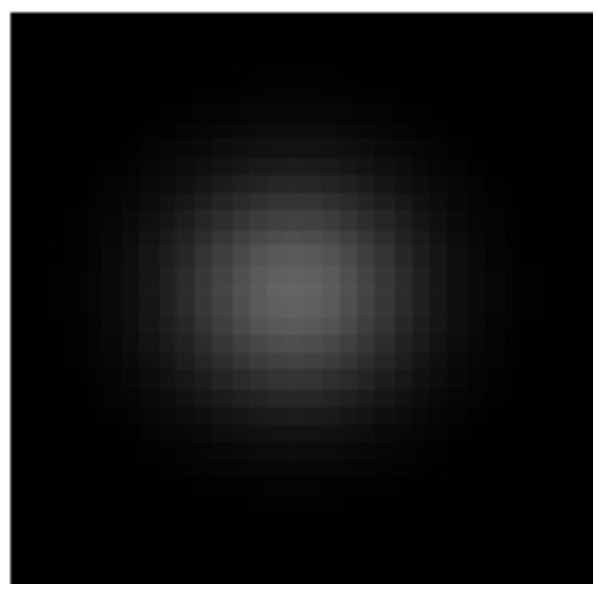

(a)

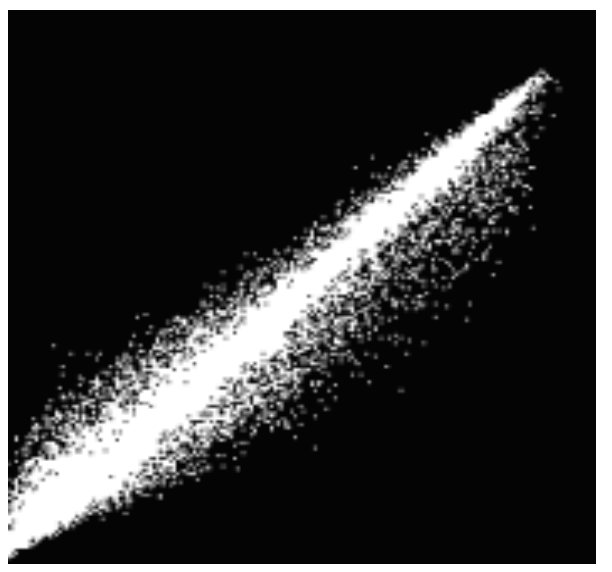

(b)

Fig. (5). (a) The image with the infrared small target, (b) the value of gray scale image center. 

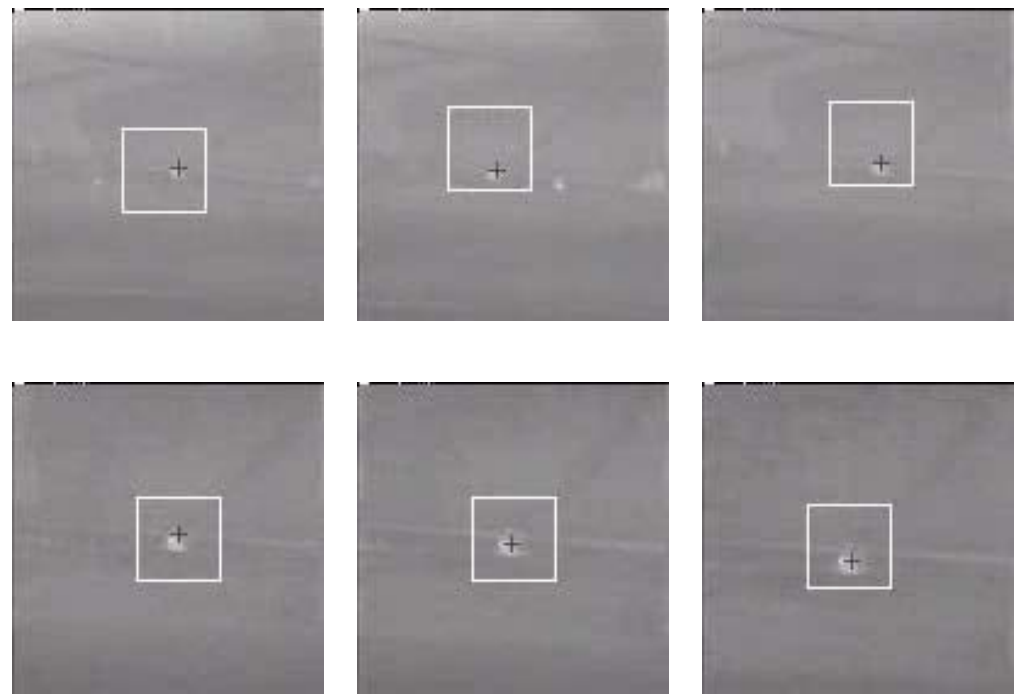

Fig. (6). The Kalman forecast tracker combined with template matching.

Table 2. The infrared small target and the value of gray scale image center.

\begin{tabular}{|l|c|c|c|c|}
\hline 73.2367 & 78.8303 & 79.5432 & 78.8303 & 73.2367 \\
\hline 82.5428 & 87.1628 & 90.0216 & 87.1628 & 82.5428 \\
\hline 87.8943 & 96.7453 & 98.2342 & 96.7453 & 87.8943 \\
\hline 92.6552 & 98.3264 & 100 & 98.3264 & 92.6552 \\
\hline 87.8943 & 96.7453 & 98.2342 & 96.7453 & 87.8943 \\
\hline 82.5428 & 87.1628 & 90.0216 & 87.1628 & 82.5428 \\
\hline 73.2367 & 78.8303 & 79.5432 & 78.8303 & 73.2367 \\
\hline
\end{tabular}

As the change situation of each pixel in the image is the basic principle of Gauss distribution background model based on Gaussian functions with Gauss, because of the Gauss model proposed for every pixel point, so it has some advantages of small target detection. Based on the Gauss function, each point in the image modeling is different, and the background modeling methods can be divided into two distribution and distribution background modeling, and background modeling.

Fig. (6) lists the Kalman forecast tracker combined with template matching algorithm for target tracking results. Here, respectively tenth, 20, 30 tracking results, 40, 50, 60, $70,80,90$ frame diagram are intercepted.

\section{CONCLUSION}

With the development of infrared imaging, infrared imaging system has been widely used in aviation and space remote sensing, night navigation, target detection and fire control, guidance and other civil and military fields. Detection and tracking technology is one of the key techniques of infrared imaging system as it uses the image processing algorithm in clutter background and the strong noise environment target automatic detection. The key is the performance of the algorithm on infrared imaging, and the intelligent de- gree of the operating distance of the system. Research on technology in the field of perfect development, new theories and new algorithms is constantly emerging. Infrared imaging of moving target detection and tracking has its special technical difficulties, as how the signal-to-noise ratio is low, the environment of complicated background, when failure occurs in the traditional detection method, and how the infrared small target can be detected accurately in real time tracking. Target feature extraction is a hot topic at this stage of research at home and abroad.

\section{CONFLICT OF INTEREST}

The author confirms that this article content has no conflict of interest.

\section{ACKNOWLEDGEMENTS}

Declared none.

\section{REFERENCES}

[1] J.X. Wu, T. Wang, Z. Y. Suo, and Z. Bao, "DOA estimation for ULA by spectral Capon rooting method", Electronics Letters, vol. 45, no. 1, pp. 84-85, 2009.

[2] J. M. Xin, and A. Sane, "Linear prediction approach to direction estimation of cyclostationary signals in multipath environment", IEEE Transactions on Signal Processing, vol. 49, no. 4, pp. 710720, 2001.

[3] E. Grosicki, K. Abed-Meraim, and K. Y. Hua, "A weighted linear prediction method for near-field source localization ", IEEE Transactions on Signal Processing, vol. 53, no. 10, pp. 3651-3660, 2005.

[4] T. B. Lavate, V. K. Kokate, and A. M. Sapkal, "Performance analysis of MUSIC and ESPRIT DOA estimation algorithms for adaptive array smart antenna in mobile communication", International Journal of Computer Networks, vol. 2, no. 3, pp. 152-158, 2010.

[5] A. Hirata, T. Morimoto, and Z. Kawasaki, "DOA estimation of ulta-wideband EM waves with MUSIC and interferometry", IEEE Antennas and Wireless Propagation Letters, vol. 2, no. 1, pp. 190193, 2003.

[6] F. Taga, "Smart Music algorithm for DOA estimation", Electronics Letters, vol. 33, no. 3, pp. 190-191, 1997.

[7] W. Sun, J. L. Bai, and K. Wang, "Novel method of ordinal bearing estimation for more sources based on obique projector", Journal of 
Systems Engineering and Electronics, vol. 20, no. 3, pp. 445-449, 2009.

[8] C. K. Man, J. Park, W. Jung, H. Kim, and Y. J. Kim, "Development of a standard communication protocol for an emergency situation management in nuclear power plants," Annals of Nuclear Energy, vol. 37, no. 6, pp. 888-893, 2010.

[9] J. Barnett, "Statistical analysis of median subtraction filtering with application to point target detection in infrared backgrounds," Proceedings of the SPIE, vol. 1050, pp. 10-15, 1989.

[10] V. T. Tom, "Morphology-based algorithm for point target detection in infrared backgrounds," In: Proceedings of the SPIE, vol. 1954, pp. 2-11, 1993.

[11] S. D. Deshpande, "Max-mean and max-median filters for detection of small-targets," Proceedings of the SPIE, vol. 3809, pp. 74-83, 1999.

[12] D. Casasent, and A. Ye, "Detection filters and algorithm fusion for ATR, IEE," Transactions on Image Processing, vol. 6, pp. 114$125,1997$.

[13] X. Wang, L. Liu, and Z.M. Tang, "Infrared dim target detection based on fractal dimension and third-order characterization," Chinese Optics Letters, vol. 7, pp. 931-933, 2009.

[14] S. G. Sun, "Target detection using local fuzzy thresholding and binary template matching in forward-looking infrared images," $\mathrm{Op}$ tical Engineering, vol. 46, no. 3, 2007.

[15] L. Itti, C. Koch, and E. Niebur, "A model of saliency-based visual attention for rapid scene analysis," IEEE Transactions on Pattern Analysis and Machine Intelligence, vol. 20, pp. 1254-1259, 1998.
[16] B. C. Ko, J. Nam, "Object-of-interset image segmentation based on human attention and semantic region clustering," Journal of the Optical Society of America, vol. 23, pp. 2462-2470, 2006.

[17] Y. Xu, Y. Zhao, C. Jin, Z. Qu, L. Liu, and X. Sun, "Salient target detection based on pseudo-Wigner-Ville distribution and Renyi entropy," Optics Letters, vol. 35, pp. 475-477, 2010.

[18] W. Li, "Saliency-based automatic target detection in forward looking infrared images," In: Proceedings IEEE Conference on Image Processing, pp. 957-960, 2009.

[19] J. Li, X. An, and X. Xu, "Visula saliency based on scale-space analysis in the frequency domain," IEEE Transactions on Pattern Analysis and Machine Intelligence, vol. 35, pp. 996-1010, 2013.

[20] M. Haralick, "Digital step edges from zero crossing of second directional derivatives," IEEE Transactions on Pattern Analysis and Machine Intelligence, vol. 6, pp. 58-68, 1984.

[21] L. Yang, J. Yang, and K. Yang, "Adaptive detection for infrared small target under sea-sky complex background," Electronics Letters, vol. 40, no. 17, pp.1083-1085, 2004.

[22] G. D. Wang, C. Y. Chen, and X. B. Shen, "Facet-based infrared small target detection method," Electronics Letters, vol. 41, no. 22, pp. 1244-1246, 2005.

[23] X. Hou, and L. Zhang, "Saliency Detection: A Spectral Residual Approach," In: Proceedings IEEE Conference on Computer Vision and Pattern Recognition, 2007.

[24] C. Guo, Q. Ma, and L. Zhang, "Spatio-Temporal Saliency Detection Using Phase Spectrum of Quaternion Fourier Transform," In: Proceedings IEEE Conference on Computer Vision and Pattern Recognition, 2008

(C) Huanhai Yang; Licensee Bentham Open

This is an open access article licensed under the terms of the (https://creativecommons.org/licenses/by/4.0/legalcode), which permits unrestricted, non-commercial use, distribution and reproduction in any medium, provided the work is properly cited. 